\title{
Differences in the Between-Person and Within-Person Structures of Affect Are a Matter of Degree
}

\author{
ANNETTE BROSE ${ }^{1,2 *}$, MANUEL C. VOELKLE ${ }^{1}$, MARTIN LÖVDÉN ${ }^{1,3}$, ULMAN LINDENBERGER $^{1}$ \\ and FLORIAN SCHMIEDEK ${ }^{1,4}$ \\ ${ }^{1}$ Center for Lifespan Psychology, Max Planck Institute for Human Development, Berlin, Germany \\ ${ }^{2}$ Research Group of Quantitative Psychology and Individual Differences, KU Leuven, Leuven, Belgium \\ ${ }^{3}$ Aging Research Center, Karolinska Institute, Stockholm, Sweden \\ ${ }^{4}$ German Institute for International Educational Research (DIPF), Frankfurt am Main, Germany
}

\begin{abstract}
This study tested whether the structure of affect observed on the basis of between-person (BP) differences is equivalent to the affect structures that organize the variability of affective states within persons (WP) over time. Further aims were to identify individual differences in the degree of divergence between the WP and BP structure and examine its association to dispositional and contextual variables (neuroticism, extraversion, well-being and stress). In 100 daily sessions, 101 younger adults rated their mood on the Positive and Negative Affect Schedule. Variability of five negative affect items across time was so low that they were excluded from the analyses. We thus worked with a modified negative affect subscale. WP affect structures diverged reliably from the BP structure, with individual differences in the degree of divergence. Differences in the WP structural characteristics and the degree of divergence could be predicted by well-being and stress. We conclude that BP and WP structures of affect are not equivalent and that BP and WP variation should be considered as distinct phenomena. It would be wrong, for example, to conceive of positive and negative affect as independent at the WP level, as suggested by BP findings. Yet, individual differences in WP structural characteristics are related to stable BP differences, and the degree to which individuals' affect structures diverge from the BP structure can provide important insights into intraindividual functioning. Copyright (C) 2014 European Association of Personality Psychology
\end{abstract}

Key words: positive and negative affect; PANAS, ergodicity, intraindividual variability; microlongitudinal design

Psychologists' knowledge about the structure underlying affective experiences is dominated by results from factor analyses using cross-sectional data. Such approaches allow the study of how different facets of affect covary across people at a given point in time (e.g., whether individuals who are more likely than others to experience anger are also more likely than others to experience nervousness). However, there are not only such between-person (BP) differences in affective experiences and BP patterns of covariation-a fundamental characteristic of affective experiences is that they change over time within persons (Kuppens, Oravecz, \& Tuerlinckx, 2010). The patterns of covariation are therefore relevant from a BP as well as a within-person (WP) perspective.

Associations among variables may or may not be equivalent within and across individuals (Borsboom, Mellenbergh, \& van Heerden, 2003; Hamaker, Nesselroade, \& Molenaar, 2007; Voelkle, Brose, Schmiedek, \& Lindenberger, in press). Unfortunately, insights from the BP level of analyses that are dominant in the literature are often (implicitly) generalized to the WP level of analysis. Such generalization is problematic because the patterns and causes of covariation can vary

*Correspondence to: Annette Brose, Research Group of Quantitative Psychology and Individual Differences, KU Leuven, Leuven, Belgium.

E-mail: annette.brose@kuleuven.be across these two levels in a meaningful way-in fact they likely do (Molenaar, 2004). It would, for example, be relevant from a clinical perspective whether patients experience all facets of negative affect simultaneously in specific situations, as would be concluded from BP structure analysis, or whether their affective disturbances are restricted to specific facets of negative affect. Hence, this study investigates whether findings on the BP structure of affect are informative on how affective states travel together across time within individuals. Our focus will be on the structures' loading patterns and factor intercorrelations.

This question and related issues have been addressed in the past. Examples are comparisons of WP and BP affect structures by means of congruency coefficients (Zevon \& Tellegen, 1982), examinations of individual differences in structural components of WP affective experiences (Feldman, 1995; Kuppens, 2008; Larsen \& Cutler, 1996; Lebo \& Nesselroade, 1978), comparisons of discrete as opposed to dimensional models of affect at the BP and WP level (Vansteelandt, Van Mechelen, \& Nezlek, 2005; Zelenski \& Larsen, 2000), and, finally, recent comparisons of the BP structure to the average WP structure using multilevel modelling (Bleidorn \& Peters, 2011; Merz \& Roesch, 2011).

Yet to date, structural equivalence has not been tested formally in the field of affect-comparisons across levels or across multiple individuals lacked statistical tests; 
comparisons are complicated by the fact that BP crosssectional analyses of affect confound trait and state variance components, which makes it difficult to interpret similarities in structures across levels of analyses (Brose, Lindenberger, \& Schmiedek, 2013); studies analysed WP structures, but not in relation to the BP structure; and whether the average WP structure resembles a single individual's WP structure remains an open question.

This study fills this gap. Using data from the COGITO study, we tested the equivalence of the BP affect structure to single individuals' WP affect structures. We departed from a two-factor model that found empirical support in crosssectional analysis of BP variation (Watson, Clark, \& Tellegen, 1988). Furthermore, this study investigates relationships between WP structural characteristics and dispositional and contextual variables (i.e., by examining the role of personality, well-being and stress for individual differences in WP structures). In this context, we hypothesized that some individuals' structures are more similar to the BP structure than that of others and that this degree of divergence would be predictable by dispositional variables.

\section{The between-person structure of affect}

In the circumplex model of affect, affective experiences are organized in a two-dimensional space, the dimensions being valence and arousal (Russell, 1980; Figure 1). Watson and Clark (1997) posit that experiences with the same valence (e.g., nervous and upset) are positively intercorrelated and that oppositely valenced states (e.g., nervous and enthusiastic) show weak correlations. Accordingly, they labelled the basic dimensions as positive and negative affect (PA and NA) and their instrument Positive and Negative Affect Schedule (PANAS; Watson \& Clark, 1997). According to a second prominent position, affect is structured by two bipolar dimensions, valence and arousal (Barrett \& Russell, 1998). A principle in line with this position is that 'the correlation between two affects is the function of the angle between them within a circular ordering' (Carroll, Yik, Russell, \& Barrett, 1999), specifically, the cosine of the angle. The PANAS is

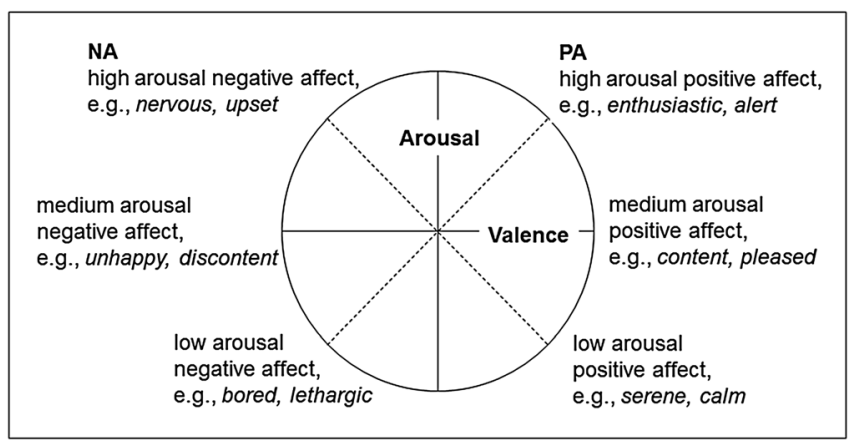

Figure 1. The affect circumplex with items clustered according to valence and high, medium and low arousal; PA and NA: positive and negative affect, respectively, the two basic dimensions according to Watson and Clark (1997) and as measured with the PANAS; according to Carroll et al. (1999), the principles formulated about the PANAS are special cases of a broader scheme; schema adapted from Carroll et al. (1999). limited to items measuring high arousal PA and NA that are $90^{\circ}$ apart $\left(\operatorname{cosine} e_{90^{\circ}}=0\right)$. Hence, according to both positions, correlations of items within the PA and NA dimension, respectively, should be positive and high, whereas correlations of items across the PA and NA dimension should be low or absent. A two-factor structure should underlie this pattern, and cross-sectional studies of trait and state affect support this proposition. The factor intercorrelation is higher in cross-sectionally administered state versions of the PANAS than in its trait version, however (Diener \& Emmons, 1984).

Hence, we propose a two-factor model for the PANAS with a simple structure and homogeneous loadings on the two factors at the BP level. As this study uses aggregated states across time for the analyses of BP differences to prevent the BP measure to be confounded with states (see, e.g., Hamaker et al., 2007), the majority of prior findings can mainly serve as proxy (i.e., cross-sectional measures confound states and traits; their structure should thus reflect a blurred state and trait structure; Brose et al., 2013). Also, allowing for a correlation between PA and NA and for residual correlations among PANAS items that originally belonged to different content domains ${ }^{1}$ was shown to improve model fit (e.g., Crawford \& Henry, 2004). These parameters are also modelled in this study.

\section{Causes of affect variation at different levels of analyses}

Having formulated an assumption about the BP structure of $\mathrm{PA}$ and NA, do we assume equivalence across levels, that is, do we expect that the underlying structure of affect measured across individuals reflects the structure of affect within individuals? To answer this question, we now elaborate on the conceptual meaning of PA and NA at the two levels. The structural characteristics that will be focused upon are the loading patterns and the factor intercorrelation.

\section{The between-person level}

At the BP level of analysis, the two latent variables PA and NA are the dispositions that are causally responsible for reporting negative and positive affective states (e.g., guilt, fear, excitement, or inspiration; see Borsboom et al., 2003). BP differences in the PA and NA true scores determine BP differences in scores of specific items. As reviewed above, PANAS items measuring PA and NA correlate strongly within each domain. Thus, relative to other individuals, a person's scores on a range of items are homogeneous, although the items may be phenomenologically different (e.g., fear and anger). Such a pattern results in homogeneous factor loadings in BP factor analysis (see Figure 2 for illustration).

The BP differences in PA and NA are the result of ontogenetic processes and are related to personality. In particular, neuroticism and extraversion differentially determine the sensitivity to negative and positive stimuli (Watson \& Clark, 1992). Pathways are temperamental (i.e., endogenous) and

${ }^{1}$ Zevon and Tellegen (1982) distinguished 20 content categories of affective experiences that cover the affective domain (e.g., anger, distress and fear); such a distinction is in line with a categorical view of affective experiences. 


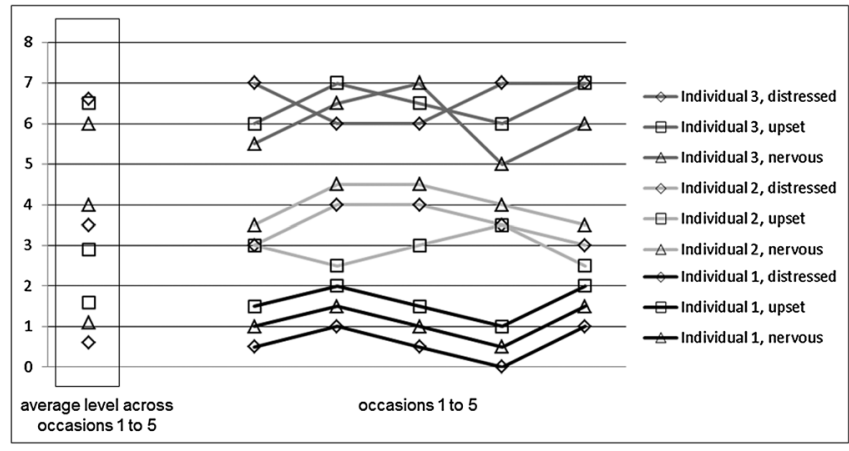

Figure 2. Illustration of between-person differences and within-person variation of affective experiences. The pattern of between-person differences scores would result in homogeneous loadings on an NA factor. The patterns of each individual's time series would result in person-specific loading patterns. In Individual 1, all aspects of NA travel together across time, which would result in a loading pattern comparable to the between-person level in terms of homogeneity across loadings. Individual 2 experiences the states distressed and upset simultaneously, and the three states seem to be independent in Individual 3. This would result in less homogeneous loading patterns in Individuals 2 and 3.

instrumental (e.g., extraverted individuals more frequently involve themselves in social events, which is associated with more frequent PA). Moreover, contextual variables such as life events or psychological illness are associated with BP differences in affect levels, in particular with NA (Watson, 1988). The causes for BP differences in trait affect differentially impact on PA and NA, and this seems to underlie the latters' independence.

\section{Moving from the between-person to within-person level} Can knowledge about BP differences in affect as described above be transferred to variation at the WP level? Will occasions with high scores on the latent variable NA (PA) be occasions with high scores across all items measuring NA (PA), and will the occurrence of NA be independent of the occurrence of PA across time?

We think neither is likely to be the case. The relationship between latent and manifest variables as described is confined to the BP perspective because the position on the latent variable is conceptualized as a constant (i.e., a trait) and hence cannot covary with a specific item across time on short timescales (Borsboom et al., 2003). Second, we argue that the causes of affect variation differ across the BP and WP level and that this renders equivalence unlikely.

\section{The within-person level}

Many of the causes of affect variation at the WP level are inherently volatile. For example, circadian rhythms, sleep quality and minor hassles (e.g., missing a bus) influence affect from hour to hour and on the timescale of days (e.g., Brose, Schmiedek, Lövdén, \& Lindenberger, 2011). Transitory shifts in life circumstances (e.g., overload at work) change affect across months (e.g., van Eck, Nicolson, \& Berkhof, 1998). It is immediately evident that many of these causes of variation elicit distinct affective states (e.g., low sleep quality may elicit sluggishness but not necessarily anger; missing a bus may elicit anger but not sadness).
In addition, affect variation within individuals across time is not merely determined by the situation but reflects an interaction between the person's disposition and the situation. For example, Individual A, a person with the disposition to feel lonely, may be sad and jittery when experiencing interpersonal tensions, whereas Individual B, a person low in agreeableness, may be upset and irritable at such times. This extends the number of possible patterns of WP covariation (number of contexts by number of dispositions, depending on the number of relevant dispositions). The structure of affect at the level of individuals should therefore be person specific (a function of the person across situations; Larsen \& Cutler, 1996; Wessman \& Ricks, 1966; Zevon \& Tellegen, 1982). The right part of Figure 2 illustrates the idea of person-specific covariation. Only Individual 1's structure of covariation resembles the BP structure of affect. Factor analysing WP affect variation would therefore likely result in more heterogeneous factor loadings on a PA and NA factor than at the BP level. Moreover, as situations that elicit positive and negative affect simultaneously are not common, individuals should less likely experience PA and NA simultaneously. Instead, PA and NA should be negatively correlated at the WP level. The latter was indeed supported by multilevel factor analysis of BP and WP affect structures (Bleidorn \& Peters, 2011; Merz \& Roesch, 2011; see also Diener \& Emmons, 1984; Schmukle, Egloff, \& Burns, 2002).

\section{Hypotheses 1}

Given that PA and NA have a different meaning and causes of variation at the BP and WP level of analysis (i.e., average behavioural tendencies related to endogenous factors versus person-specific person-environment interactions), we expected that prior insights on the $\mathrm{BP}$ affect structure may not be informative on the structure of affect variation within individuals across time. For WP affect variation, we expected (1) individual differences in the structural characteristics of WP affect variation, in particular, in the loading patterns and the PA-NA factor intercorrelation. On average, the factor loadings should be less homogeneous, and the factor intercorrelation should be smaller than at the BP level. For these reasons, we hypothesized (2) that the majority of individuals' affect structures would not resemble the BP structure. A direct test of this hypothesis has not yet been conducted. Here, we applied an innovative approach to conduct this test (Voelkle, Oud, von Oertzen, \& Lindenberger, 2012).

\section{Affect variation: relationships between the two levels of analyses}

The line of reasoning summarized above suggests that BP and WP affect structures are regulated by different sets of antecedents. Does this warrant the conclusion that insights about BP differences in affect are useless for understanding WP affect structures? In our view, this conclusion would be wrong. It would miss out on an entire field of research, namely on the question which factors make an individual's affect structure more or less similar to the BP structure. There may be differences in the degree to which individuals' 
structures depart from the BP structure. Previous research has revealed individual differences in WP structural characteristics (e.g., the PA-NA factor intercorrelation and the number of extracted factors in exploratory factor analyses). The latter are related to stable BP differences (e.g., resilience and neuroticism; Carstensen, Mayr, Pasupathi \& Nesselroade, 2000; Ong $\&$ Bergeman, 2004). These findings indicate interdependence between dispositions and processes in the field of affect (for discussions on this interdependence in the field of personality, see Mischel \& Shoda, 1998). In the following, we will elaborate on the interdependence of levels analysis and on substantive reasons that may result in WP structures that are relatively equivalent to the BP structure, and we will discuss the degree of BP-WP structural divergence (cf. Voelkle et al., in press).

\section{Negative affect loading pattern}

First, individuals whose NA factor structure resembles the BP NA factor structure (i.e., homogeneous loadings) should have responses to affect-inducing stimuli that are not very differentiated - a threat should be associated with a general affective response (e.g., including fear, anger and nervousness) in these individuals, and discriminating features of situations seem less relevant. Such a response pattern may be typical for individuals high in neuroticism as they may have a lower threshold for affective states to be activated given their sensitivity to emotional material (Watson \& Clark, 1992). Likewise, such a response pattern may be typical in individuals with high stress levels because their ability to differentiate between situations and affective states is known to be reduced-a stimulus is either good or bad and nuances lose importance (Zautra, Berkhof, \& Nicolson, 2002). Positive emotions and psychological resilience have the opposite effect on how negative affective states are experienced. Psychological well-being has an ameliorating effect on negative affective experiences (Ong, Bergeman, Bisconti, $\&$ Wallace, 2006). We propose that this may result in a specific reaction to a stressor as opposed to a generalized increase in multiple NA facets and therefore may result in an NA factor that is less similar to the BP factor (i.e., a more differentiated NA factor).

\section{Positive affect loading pattern}

Individuals whose PA factor resembles the BP PA factor (i.e., homogeneous loadings) should have a less differentiated response to affect-inducing positive stimuli than individuals with heterogeneous loadings. This may occur in individuals with high levels of extraversion because of a proneness to experience positive emotions (Watson \& Clark, 1992). Moreover, people differ regarding their focus on valence or arousal aspects of affect (Feldman, 1995). Therefore, it seems likely that those individuals who focus more on arousal aspects of affective experiences have PA loading patterns similar to the BP PA loading pattern when PA is measured with the PANAS, because the PANAS captures high arousal states only.

\section{Positive-negative affect factor intercorrelation}

Third, individuals who have a PA-NA factor intercorrelation that is similar to the BP factor intercorrelation (a nonsignificant association) should be those who manage to keep these two dimensions separate, irrespective of whether a situation is threatening or rewarding. This capacity is referred to as emotional complexity, poignancy and cooccurrence (Carstensen et al., 2000; Ong \& Bergeman, 2004). It is positively correlated with indicators of resilience and negatively correlated with sustained negative affect and neuroticism (Ong \& Bergeman, 2004). Over and above this, the ability to differentiate between PA and NA is a function of stress (Zautra et al., 2002). Stress exposure is resource demanding. Because differentiating between different emotionally relevant aspects of situations also requires resources, a stressful period is supposed to constrain the former, resulting in more negative PA-NA associations.

\section{Hypotheses 2}

We expected (3) that the degree to which the WP structures deviate from the BP structure are reflected in individual differences in WP structural characteristics (loading patterns, the latent correlation). Resemblance to the BP structure was expected to decrease with more heterogeneous PA and NA loading patterns and more negative correlations between PA and NA. Moreover, we hypothesized (4) that BP differences in WP structural characteristics (in the homogeneity of the PA and NA loading patterns and the factor intercorrelation) can be predicted by dispositional and contextual variables. More homogeneous NA loadings should be related to higher levels in neuroticism and stress, and to lower levels in well-being. More homogeneous PA loadings should be related to higher levels in extraversion and arousal focus. The correlation between PA and NA should be positively associated with well-being and negatively associated with neuroticism and stress. Emerging from the preceding, and finally, we explored whether (5) the degree of nonequivalence/divergence between WP and BP affect structures is substantively meaningful. Building upon Hypothesis 4, we examined whether individuals high in neuroticism and stress and low in well-being show WP structures that are more equivalent to the BP structure because their NA items tend to travel in time together (i.e., homogeneous NA loadings). At the same time, we surmised that individuals high in neuroticism, stress and low in well-being show WP structures that are less equivalent to the BP structure because their factor intercorrelation would be more negative than observed at the BP level. We will introduce a statistical approach that is capable of disentangling these divergent hypotheses. Finally, we expected that individuals with higher levels of extraversion and arousal focus show WP structures that resemble the $\mathrm{BP}$ structure, reflecting similarities in $\mathrm{PA}$ loading patterns.

\section{METHOD}

The current investigation is part of a larger study, the COGITO study, conducted at the Center for Lifespan Psychology, Max Planck Institute for Human Development, Berlin (for details, see Schmiedek, Bauer, Lövdén, Brose, \& Lindenberger, 2010). The COGITO study consisted of younger and older adults; it followed a pretest-posttest control group design with 
a microlongitudinal phase of about 100 days in the experimental group. This study focuses on this phase.

\section{Participants and procedure}

The sample consisted of the entire subsample of 101 younger adults (51.5\% women; age: $20-31, M=25.6, S D=2.7)$. Participants were recruited through newspaper advertisements, wordof-mouth recommendations and flyers circulated in Berlin, Germany. In the microlongitudinal study phase (1-1.5 hours each), the total number of sessions per person ranged from 87 to $107(M=101)$. This phase was completed in 23 weeks (mean number of days $=158$, median number of days $=148$ ). Incentives for study participation varied between $€ 1,450$,- and $€ 1,950$,-, depending on the pace of study completion. The daily sessions were carried out from Monday to Saturday between 8 AM and 7.30 PM. Participants worked on computerized cognitive tasks and self-report measures individually in rooms with three to six work places.

\section{Measures}

\section{Positive and negative affect}

To assess positive and negative affect across all study days, the state version of the PANAS (Watson et al., 1988) was applied. On each day, subjects rated their momentary affect on 20 items using an 8-point scale $(0=$ does not apply at all, $7=$ applies very well). State scores, which were used for analyses at the WP level, were deviations from average tendencies. For the analysis of the BP level, we used the average tendencies in affective experiences across study time (i.e., the WP means). With this decision, we follow the reasoning by Hamaker et al. (2007) according to whom any observation at a single occasion is the sum of a trait and a state component. In a stationary time series, the mean of the observed scores across many occasions is the trait in the sense of an average behavioural tendency; the average of the states should be zero. Such an operationalization of trait affect is advantageous in comparison to commonly used cross-sectional assessment of trait affect in that states and traits are not confounded. Prior to further analysis, the affect items were inspected for variability. Some NA items showed no variance within 41 individuals across study time (guilty, $8 \%$ of the participants; scared, $16 \%$ of the participants; hostile, $10 \%$ of the participants; ashamed, $8 \%$ of the participants; and afraid: $8 \%$ of the participants). These five items also showed reduced variability in comparison to the other NA items in the remaining 60 individuals: their range of within-person variances was 0.81 to $1.02, M=0.93$; in the remaining items, the range of the within-person variance was 1.26 to $1.73, M=1.48$. Because variability is necessary for the following covariance structure analyses, items with little variance were excluded from further analysis. Please note that this implies a modification of the latent construct under investigation - the NA subscale of this study has a narrower scope than the original NA subscale of the PANAS.

\section{Between-person differences variables}

In this study, dispositional and stable contextual variables (i.e., neuroticism, extraversion, well-being and stress) were investigated as correlates of how individuals' affective states covary across time and of the degree to which individuals' structures adhere to the BP structure (e.g., whether individuals high in neuroticism show WP structures that are relatively alike to the BP structure of affect). Unless stated differently, BP differences variables were assessed at pretest. To determine individuals' positions on BP difference variables, factor scores were estimated.

The personality factors neuroticism and extraversion were each measured with 48 items from the German version of the Revised NEO Personality Inventory (Costa \& McCrae, 1992; Ostendorf \& Angleitner, 2004). To measure focus on arousal when experiencing affect, we used items of the Affect Intensity Measure (AIM; Larsen, Diener, \& Emmons, 1986). In particular, we identified a target item that reflects an arousal focus during emotional episodes (the AIM item 'When I am happy, I feel very energetic'). By means of an exploratory factor analysis, we then identified items loading on the same factor. In a third step, we estimated factor scores for this factor.

Three aspects of well-being were assessed, an affective and cognitive component and the six facets of psychological wellbeing that are being distinguished in the multidimensional model of well-being (Ryff, 1989). These six facets are autonomy, environmental mastery, personal growth, positive relations with others, purpose in life, and self-acceptance. They were assessed with three items each. They emerged in discussions on the nature of well-being across subfields of psychology (e.g., developmental and clinical psychology; Ryff, 1989). In the COGITO study participants rated how well each item describes them. Life satisfaction reflects cognitive well-being and was assessed with the Satisfaction With Life Scale (SWLS; Diener, Emmons, Larsen, \& Griffin, 1985). Answers were provided on the 8-point scale just described. Affective well-being was assessed by means of the PANAS (Watson et al., 1988). Participants rated how often they had experienced the $20 \mathrm{PA}$ and NA states throughout the last year. Affective well-being was also assessed with the German version of the Center for Epidemiologic Studies-Depression Scale (CES-D; Hautzinger, 1988). Individuals evaluated how often they felt in accordance with the items throughout the last week.

Overall stress level throughout the study was assessed with four indicators. First, major negative life events were measured with items from the Social Readjustment Rating Scale (SRRS; Hobson et al., 1998). Additionally, an open question was posed on each day of the microlongitudinal phase for participants to report events. If such an event fell into the SRRS, it was also coded as a major life event (e.g., loss of a partner). Second, the number of reported minor stressful events across the microlongitudinal phase was counted. Third, individuals rated their level of perceived stress on each of the 100 occasions on three items by means of an adapted version of the Perceived Stress Scale (PSS; Cohen, Kamarck, \& Mermelstein, 1983). The scores on each item were averaged across the 100 occasions. Next, factor scores were estimated using these perceived stress aggregates.

\section{Statistical analyses}

Data preprocessing and preliminary analyses

Data were preprocessed before the study aims were approached. First, we adjusted for linear, within-person trends 
in affective experiences across study time. This was done per item and person by means of multilevel random effects models. ${ }^{2}$ Second, the data were $\mathrm{z}$-transformed at the WP level (per item and person) and at the BP level (per item). This decision (i.e., to use correlation matrices) is based on the fact that seminal work on the structure of affect, which is also cited in this paper, used correlation matrices and because this study is concerned with the question to what extent prior findings on the BP affect structure can tell us something about within-person functioning. Thus, we think it is necessary to present results that are comparable with those in the literature. To estimate the overall plausibility of the factor structure at both levels of analysis, especially given the present deviations from the common PANAS due to item exclusion, we performed a two-level confirmatory factor analysis imposing configural invariance using Mplus $^{3}$. The path diagram in Figure 3, Model 1, resembles the specified model, with the essential feature that a BP model and a WP model are estimated simultaneously (Muthén, 1994).

\section{Between-person versus within-person affect structures} (Hypothesis 1 and 2)

To model the structure of affect at the BP level (Figure 3, Model 1), a confirmatory factor analysis (CFA) was carried out on the BP part of the data. The model is defined as shown in Equation 1a (please see Figure 3, Model 1):

$$
\mathrm{y}(i)=\Lambda \eta(i)+\varepsilon(i)
$$

where $\mathrm{y}(i)$ is a 15 -variate vector of observations for individuals, $i=1,2, \ldots, N, \Lambda$ is a $15 \times 2$ factor loading matrix of the two latent factors PA and NA $(\eta(i))$, and $\varepsilon(i)$ is a 15 -variate residual (specific error + measurement error) vector. The two factors were allowed to correlate without crossloadings. Factor variances were fixed to 1 , and error correlations were allowed between items originating from the same content domain (see above). The analyses were carried out in mkfm6, a Fortran program that uses Kalman filtering to estimate latent states (Dolan, 2010). This program was used to stay in the same modelling environment for the analyses that follow.

To model the structure of affect at the WP level, dynamic factor analysis (DFA) was carried out separately for each individual using mkfm6. DFA models sequential dependencies (see Figure 3, Model 2) and thus models process in the sense that present states predict future states. Modelling sequential dependencies is an adequate representation of time series

\footnotetext{
${ }^{2}$ Using session number as a predictor of the specific affective states in multilevel models (separately for each item; including fixed and random effects) revealed the presence of linear trends and random variation around fixed effects. Models with and without the linear trend were compared based on the Bayesian Information Criterion [BIC], and the model with the smaller BIC was chosen for the purpose of detrending. Trends were removed because we were interested in the structure of day-to-day variability and not in the structure of some change process that may have occurred during study time for idiosyncratic (e.g., exposure to a major stressor) or study-related reason (e.g., becoming bored of the study).
}

data (Molenaar, 1985). The model as described by Equation $1 \mathrm{~b}$ was used, residual correlations were allowed analogous to the BP model, and additionally, regressions among the latent states were introduced,

$$
\begin{gathered}
\mathrm{y}(t)=\Lambda \eta(t)+\varepsilon(t) \\
\eta(t)=\mathrm{B} \eta(t-1)+\zeta(t)
\end{gathered}
$$

Here, $\mathrm{y}(t)$ is a 15 -variate vector of observations for time points, $t=1,2, \ldots, T, \Lambda$ is a $15 \times 2$ factor loading matrix of the two latent factors PA and NA $(\eta(t))$, and $\varepsilon(t)$ is a 15variate residual (specific error + measurement error) vector. The two-variate latent state vector $\eta(t)$ is a function of the prior latent state, $\eta(t-1)$ weighted by a $2 \times 2$ matrix $\mathrm{B}$, which contains the autoregressive effects in the main diagonal. Present time 'disturbances' are then introduced as a two-variate set of latent innovations, $\zeta(t)$ (for further details, see Nesselroade, McArdle, Aggen, \& Meyers, 2002). The time series analyses were carried out with daily intervals between measurement occasions, with missing values for each calendar day without a session. Parameters of the DFA were assumed to be time invariant (i.e., it was assumed that during study participation, no major changes occurred in the participants' lives that may lead to a change in their affective dynamics).

Testing invariance across levels of analysis (Hypothesis 2) To test whether the WP affect structures differed from the BP structure, two successive multigroup models were carried out in mkfm6 for each within-between comparison (see Voelkle et al., 2012, for details). The advantage of this modelling approach to multilevel CFA is that serial dependencies at the within-person level can be modelled (i.e., the within-part of the model is a DFA). In the first multigroup model, the BP model (Figure 3, Model 1) and the WP model (the DFA model; Figure 3, Model 2) were estimated simultaneously, and all WP and BP parameters were freely estimated (all parameters as indicated by single-headed and double-headed arrows in Figure 3). This results in coefficients identical to those resulting from separate analyses of Model 1 and Model 2. In the second multigroup model, the factor loadings and the latent correlation were constrained to equality at the WP and BP level (parameters with equal signs in Figure 3, Model 1 and Model 2). This results in a different number of degrees of freedom for Multigroup Model 1 and Multigroup Model 2 and a different model fit (i.e., the likelihood). By means of a likelihood ratio test, it becomes possible to test whether the constraints resulted in a significant reduction in model fit. That is, the test reveals whether an individual's WP model significantly differs from the BP model. This procedure was repeated for all subjects.

\section{Stable dispositions and the degree of divergence (Hypotheses 3-5)}

Correlational analyses were used to analyse the relationships between WP structural characteristics and stable dispositions. 

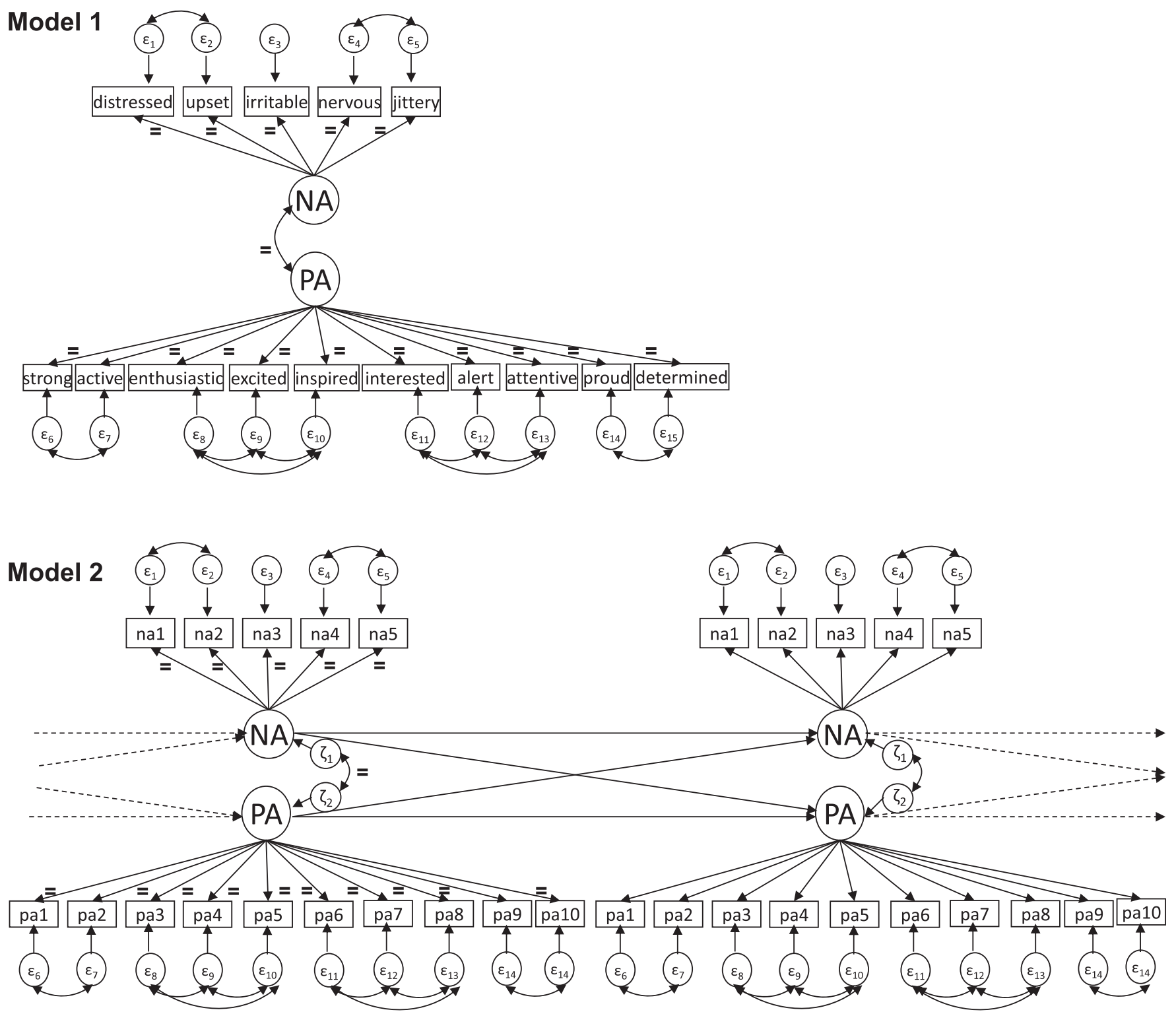

Figure 3. The BP factor model (Model 1) and the WP dynamic factor model (Model 2); na1 to na5 are the indicators distressed, upset, irritable, nervous, and jittery; pa1 to pa10 are the indicators strong, active, enthusiastic, excited, inspired, interested, alert, attentive, proud, and determined; NA and PA are the factors positive and negative affect, respectively; = refers to those parts of the two models that were constrained to be equal in the multigroup models that tested equivalence across the BP and WP level.

The relationships between the degree of divergence of WP and BP structures and stable dispositions were also approached with correlational analyses.

\section{RESULTS}

Table 1 provides descriptive information on the intraindividual means (iM) of the aggregated state PANAS and fluctuations around these $\mathrm{i} M \mathrm{~s}$, as indicated by the intraindividual standard deviations (iSD). The overall plausibility of the factor structure at the BP and WP level of analysis was examined with a two-level CFA. At this stage, all parameters were estimated without constraints across levels. Results are presented in Table 2, columns 1 and 2. The fit was acceptable, with the comparative fit index $(\mathrm{CFI})=.94$, root mean square error of approximation (RMSEA) $=.04$ and standardized root mean square residuals (SRMR) for within $=.05$ and for between $=.09$. Thus, we continued with this model in the subsequent analyses.

\section{Between-person versus within-person affect structures (Hypotheses 1 and 2)}

The between-person structure

We proposed a BP affect structure in accordance with the literature (Figure 3, Model 1). Table 2, column 3, provides the results of this analysis. As expected, the factor loadings of the NA and PA factor were relatively high, 
Table 1. Descriptive information on study variables: means and standard deviations of intraindividual means (iM) and intraindividual standard deviations (iSD) of the aggregated state PANAS

\begin{tabular}{|c|c|c|c|c|}
\hline & \multicolumn{4}{|c|}{ Within-person coefficients } \\
\hline & \multicolumn{2}{|c|}{$\mathrm{i} M$} & \multicolumn{2}{|c|}{$\mathrm{i} S D$} \\
\hline & $M$ & $S D$ & $M$ & $S D$ \\
\hline \multicolumn{5}{|c|}{ Negative affect } \\
\hline Distressed & 1.59 & 1.03 & 1.14 & 0.37 \\
\hline Upset & 1.16 & 0.90 & 1.11 & 0.38 \\
\hline Irritated & 1.44 & 1.01 & 0.99 & 0.38 \\
\hline Nervous & 1.38 & 0.99 & 0.95 & 0.35 \\
\hline Jittery & 1.74 & 1.10 & 1.01 & 0.33 \\
\hline \multicolumn{5}{|l|}{ Positive affect } \\
\hline Strong & 3.45 & 1.24 & 0.90 & 0.28 \\
\hline Active & 3.61 & 1.02 & 0.93 & 0.28 \\
\hline Enthusiastic & 2.52 & 1.16 & 1.06 & 0.34 \\
\hline Excited & 3.12 & 1.25 & 1.02 & 0.32 \\
\hline Inspired & 2.59 & 1.15 & 1.03 & 0.31 \\
\hline Interested & 3.26 & 1.20 & 0.87 & 0.28 \\
\hline Alert & 3.65 & 0.87 & 0.99 & 0.28 \\
\hline Determined & 3.51 & 1.16 & 0.99 & 0.32 \\
\hline Attentive & 3.50 & 0.95 & 0.86 & 0.25 \\
\hline
\end{tabular}

Note: The WP means of the aggregated state Positive and Negative Affect Schedule were relevant for between-person factor analysis; iSD of residuals after detrending (adjustment for linear trends).

Table 2. Results from between-person and within-person (multilevel and dynamic) factor analyses

\begin{tabular}{|c|c|c|c|c|c|}
\hline & \multicolumn{2}{|c|}{ Results multilevel CFA } & \multicolumn{3}{|c|}{ Results mkfm6 } \\
\hline & \multirow[t]{2}{*}{$\begin{array}{c}\text { Between- } \\
\text { person } \\
\text { level }\end{array}$} & \multirow[t]{2}{*}{$\begin{array}{l}\text { Within- } \\
\text { person } \\
\text { level }\end{array}$} & \multirow[t]{2}{*}{$\begin{array}{c}\text { Between- } \\
\text { person } \\
\text { level }\end{array}$} & \multicolumn{2}{|c|}{$\begin{array}{c}\text { Within- } \\
\text { person } \\
\text { level }\end{array}$} \\
\hline & & & & $M$ & $S D$ \\
\hline \multicolumn{6}{|l|}{ Loadings } \\
\hline Distressed & .84 & .59 & .83 & .52 & .27 \\
\hline Upset & .93 & .71 & .93 & .58 & .23 \\
\hline Irritated & .88 & .65 & .90 & .57 & .22 \\
\hline Nervous & .83 & .34 & .81 & .29 & .22 \\
\hline Jittery & .83 & .37 & .82 & .32 & .20 \\
\hline Strong & .80 & .55 & .81 & .48 & .15 \\
\hline Active & .82 & .53 & .82 & .46 & .16 \\
\hline Enthusiastic & .89 & .63 & .89 & .57 & .18 \\
\hline Excited & .89 & .61 & .88 & .53 & .16 \\
\hline Proud & .71 & .46 & .71 & .42 & .20 \\
\hline Inspired & .64 & .57 & .65 & .49 & .19 \\
\hline Interested & .73 & .51 & .73 & .45 & .20 \\
\hline Alert & .93 & .58 & .93 & .50 & .15 \\
\hline Determined & .80 & .59 & .80 & .51 & .17 \\
\hline Attentive & .79 & .67 & .80 & .58 & .18 \\
\hline Correlation $^{\dagger}$ & .11 & -.28 & .13 & & \\
\hline Correlation $^{\dagger \dagger}$ & & & & -.36 & .31 \\
\hline \multicolumn{6}{|c|}{ Latent regression } \\
\hline$\beta_{\mathrm{NA}}$ & & & & .29 & .34 \\
\hline$\beta_{\mathrm{NA}-\mathrm{PA}}$ & & & & .04 & .26 \\
\hline$\beta_{\mathrm{PA}-\mathrm{NA}}$ & & & & .05 & .24 \\
\hline$\beta_{\mathrm{PA}}$ & & & & .30 & .30 \\
\hline
\end{tabular}

Note: $* p<.05$; ${ }^{\dagger}$ factor intercorrelation; ${ }^{\dagger \dagger}$ correlation of innovations; $\mathrm{CFA}=$ confirmatory factor analysis; NA, negative affect; PA, positive affect. homogeneous and almost identical to the loadings of the multilevel FA. ${ }^{3}$ In line with former research, the correlation between the factors did not differ from zero, $p>.05$. Homogeneity of the NA and PA loading patterns was quantified by the $S D$ of the amount of variance explained by the factors across loadings (i.e., the $S D$ of the squared standardized factor loadings). This homogeneity index of NA loadings was 0.09 ; the homogeneity index of PA loadings was 0.14 .

\section{The within-person structures}

We expected individual differences in WP affect structures and, on average, less homogeneous PA and NA loadings and more negative correlations between the PA and NA factor. Results from single-subject DFA are summarized in Table 2 and Figure 4. Moreover, the structures of three individuals are presented in Figure 5 (they were chosen for reasons explained below). A proper solution could not be obtained for five individuals (models did not converge). Moreover, four individuals' solutions had to be excluded because the items did not form factors. ${ }^{4}$ Therefore, the following findings are based on 92 individuals' solutions.

The size of the items' loadings (averaged across all individuals' coefficients) ranged from .29 to .58 . On average, the factors were negatively and moderately correlated. All parameters showed sizeable variation across participants. The indicators of differential affective responding (i.e., the homogeneity index of the NA and PA factors) were calculated analogous to the BP level, separately for each individual and the two factors. Particularly the NA loadings appeared less homogeneous at the WP level than at the BP level. On average, the homogeneity index of NA factor was 0.22 ( $S D$ of this $S D=.12$, range $=.05$ to .53 ). The homogeneity index of the PA factor was 0.13 ( $S D$ of this $S D=.05$, range $=.06$ to .30 ). These ranges indicate that for some individuals, the nature of the factor is mainly determined by some of the items (less homogeneous pattern) whereas for others, it seems to have a broader scope (more homogeneous

${ }^{3}$ The BP model of the multilevel CFA was estimated using the BP correlation matrix of individual's means across study time. The same data were used to model the BP structure in mkfm6. Thus, the results should be highly similar. The WP model of the multilevel CFA was estimated using the average WP correlation matrix; each item was z-transformed per person before the analysis and was added to the $\mathrm{z}$-transformed WP mean. This model differs from the DFA models that were estimated separately for each individual in that the latter accounts for sequential dependencies and in that the WP structure is determined across all individuals simultaneously. Therefore, results from the WP part of the multilevel CFA may differ from the average WP coefficients of the DFAs presented in Table 2.

${ }^{4}$ In two individuals, all PA loadings were smaller than .15; in one individual, all NA loadings were smaller than .15, and in one individual, one NA loading was 1.0 whereas the others were below .15. Those nine individuals (55\% female, mean age $=27.1$ years) for whom no proper solution was obtained differed from the remaining 92 individuals in that they had lower mean levels of state negative affect across study time, $M_{92 \text { individuals }}=1.38$, $M_{9 \text { individuals }}=.72, p=.04, d=.72$. They did not differ on any of the other variables that were relevant and analyzed in this study. The effect sizes of the mean differences were small (neuroticism, extraversion, trait NA, trait PA, CESD, life satisfaction, autonomy, environmental mastery, personal growth, relations with others, purpose in life) to medium (affect intensity, major life events, daily stressors, perceived stress, average PA across 100 occasions; the nine individuals without proper solutions had lower values on these measures). This pattern is somewhat inconclusive. 




Figure 4. Distributions of within-person factor correlations (latent $r$ ) and within-person factor loadings across 92 individuals; na1 to na5: distressed, upset, irritable, nervous, and jittery; pa1 to pa10: strong, active, enthusiastic, excited, inspired, interested, alert, attentive, proud, determined; distributions of standard deviations (SD) across NA loadings and PA loadings, respectively, per individual; crosses mark the coefficients from the BP person affect structure.

pattern). The correlation between PA and NA was, on average, negative within individuals. Regarding individuals' factor intercorrelations, $62 \%$ were negative and significant, $2 \%$ were positive and significant and $36 \%$ were not different from zero. In sum, the deviations of WP results from findings at the BP level seem substantial. An empirical test of this impression will follow below.

Given this study's interest in structural characteristics that can be compared across the BP and WP level of analysis, we only briefly point to the time-dependent aspects of the WP DFA. Information on latent lagged and cross-lagged regression coefficients is reported in Table 2.

\section{Testing invariance across levels of analysis}

To test the hypothesis that individuals' structures differ from the BP structure (Hypothesis 2), two successive multigroup models were carried out for each withinbetween comparison. These comparisons were made separately for each individual. In both multigroup models, the BP structure and one WP structure were estimated simultaneously (Figure 3, Models 1 and 2). In the first multigroup model, all parameters of the WP and the BP model were freely estimated. In the second multigroup model, the factor loadings and the latent correlation were constrained to equality across groups. The change in degrees of freedom was 16 , accordingly the critical $\chi^{2}$ value $\left(\chi^{2}\right.$ crit.) of the likelihood ratio test for the subsequent model comparison is 26.3 for an alpha level of $5 \%$.

Results are presented in Figure 6. The difference in model fit $(\Delta-2 L L$, the likelihood ratio, LR) was significant in all individuals. Thus, the BP structure of trait affect was not equivalent to affect covariation within single individuals across time. Yet, there were individual differences in the degree of deviation from the BP structure as is discernible in the LR test (Figure 6). The three solutions presented in Figure 5 were chosen to demonstrate individual differences in the LR of the within-between comparisons. The WP structures of individuals with small LRs are not identical to the BP structure but seem quite similar. Others have features that more clearly differentiate them from the BP structure (e.g., the NA-PA correlation of Individual 2, the loadings of Individual 3).

\section{Stable dispositions and the degree of invariance (Hypotheses 3-5)}

Associations between within-person characteristics and the degree of invariance

We expected that the degree to which the WP structures deviate from the BP structure (i.e., the LR) is related to individual differences in WP structural characteristics (Hypothesis 3). In line with these expectations, individual differences in the LR were inversely related to the homogeneity index of NA loadings $(r=.26, p=.02)$. Individuals with more homogeneous NA loadings were more similar to the BP structure (i.e., they had a smaller LR). Likewise, the LR was inversely related to the homogeneity index across PA loadings $(r=.47, p<.0001)$. Individuals with more homogeneous PA loadings were again more similar to the BP structure. Finally, the LR was negatively related to the factor intercorrelation, $r=-.26, p=.01$. Individuals with a larger (i.e., less negative) correlation between the two factors were more alike to the BP structure. In sum, the degree of divergence was significantly related to WP structural characteristics.

\section{Associations between within-person characteristics and dispositions}

Inspired by previous research on WP affect structures, we hypothesized that differences in WP structural characteristics are associated with dispositional and contextual variables (indicators of personality, well-being and enduring levels of stress; Hypothesis 4). The correlations between the NA and PA homogeneity indices were partial correlations, adjusting for the means across the loadings, to obtain associations that merely reflect covariation across time.

Homogeneity of NA loadings (Table 3, column 1). The sign of the partial correlation between neuroticism and the homogeneity index of NA loadings pointed in the hypothesized direction but was not significant. Furthermore, we expected more heterogeneous loadings in individuals with higher well-being. In line with this expectation, higher trait PA was associated with more heterogeneous loadings. Individuals with relatively high levels of PA have a more differentiated NA factor. Likewise, higher sense of autonomy, personal growth and purpose in life were associated with more heterogeneous loadings. In contrast, and as expected, higher levels of depressive symptoms were associated with more homogeneous loadings. Indicators of enduring stress were not associated with the index of homogeneity of the NA factor. There was a trend for stressful major life events and perceived stress across study time. 


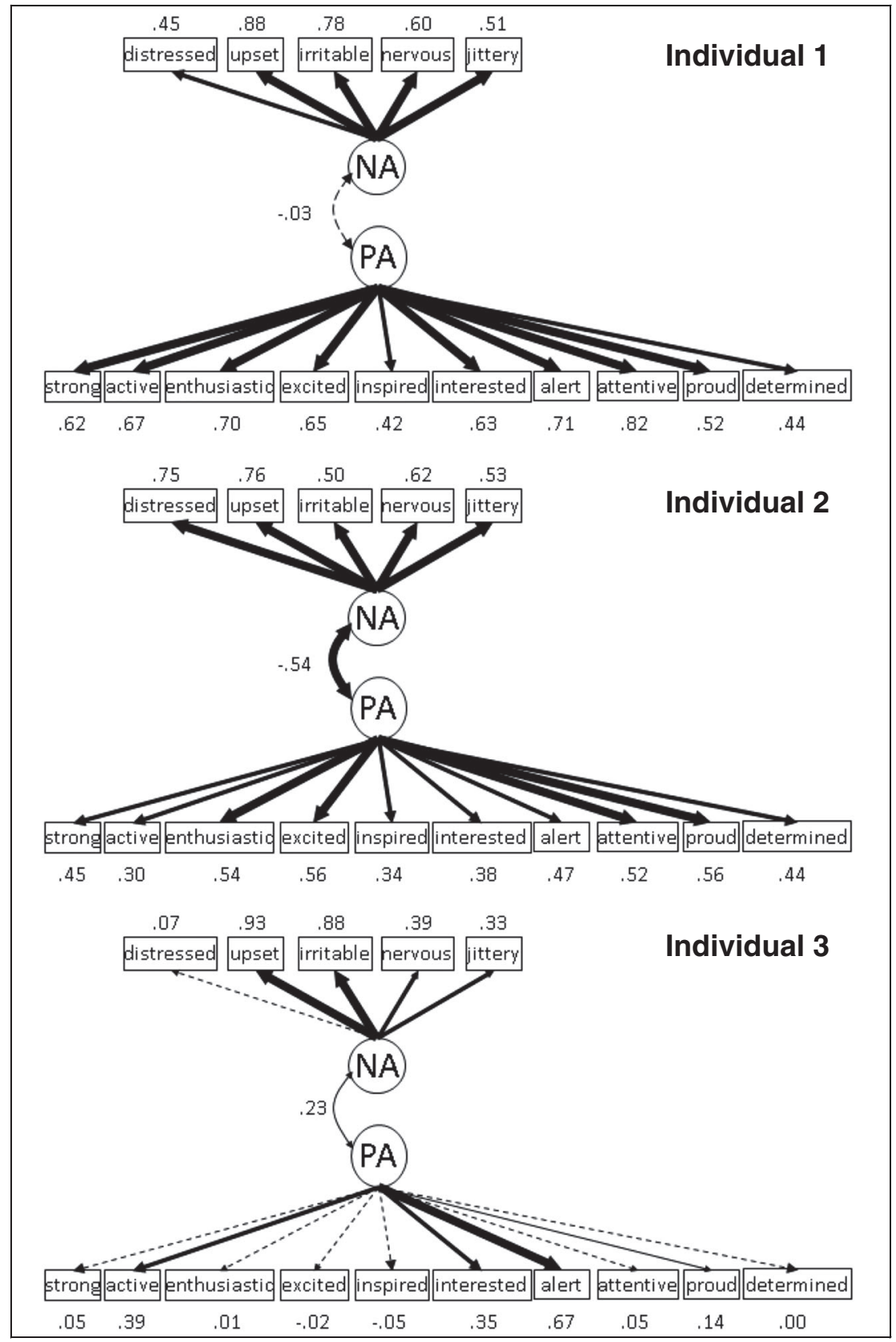

Figure 5. Solutions from dynamic factor analyses (DFA) of three individuals: Individuals 1, 2 and 3; numbers refer to the loadings and the factor intercorrelations; the choice of these individuals was based on the rank order of likelihood ratios presented in Figure 6; they have a likelihood ratio of 29.3, 75 and 214.4.

Homogeneity of PA loadings (Table 3, column 2). We had two hypotheses regarding homogeneity of the PA factor loadings, namely that individuals high in extraversion and high in arousal focus have more homogeneous PA loadings, indicating a more generalized PA response. This expectation was not supported by our findings, as the homogeneity index of the PA factor was not associated with extraversion and arousal focus.

The positive-negative affect factor intercorrelation (Table 3, column 3). Regarding the PA-NA factor intercorrelation and stable dispositions, we expected a more negative correlation in individuals high in neuroticism. The corresponding correlation did not differ significantly from zero, however. In line with expectations, indicators of well-being were associated with the PA-NA factor intercorrelation. Individuals relatively high in trait PA had correlation coefficients relatively close to zero, whereas the average of the within-person correlation was more negative (i.e., -.36). This means that these individuals with higher trait PA are more likely to experience PA and NA simultaneously at a given occasion. Likewise, individuals high in personal growth had a less negative correlation between the two factors, and a relatively high sense of environmental mastery was marginally related to the PA-NA correlation. One indicator of stress was associated with the PA-NA factor intercorrelation. Confirming our expectations, individuals who were exposed to a major stressor during the study had a more negative correlation between the factors. 


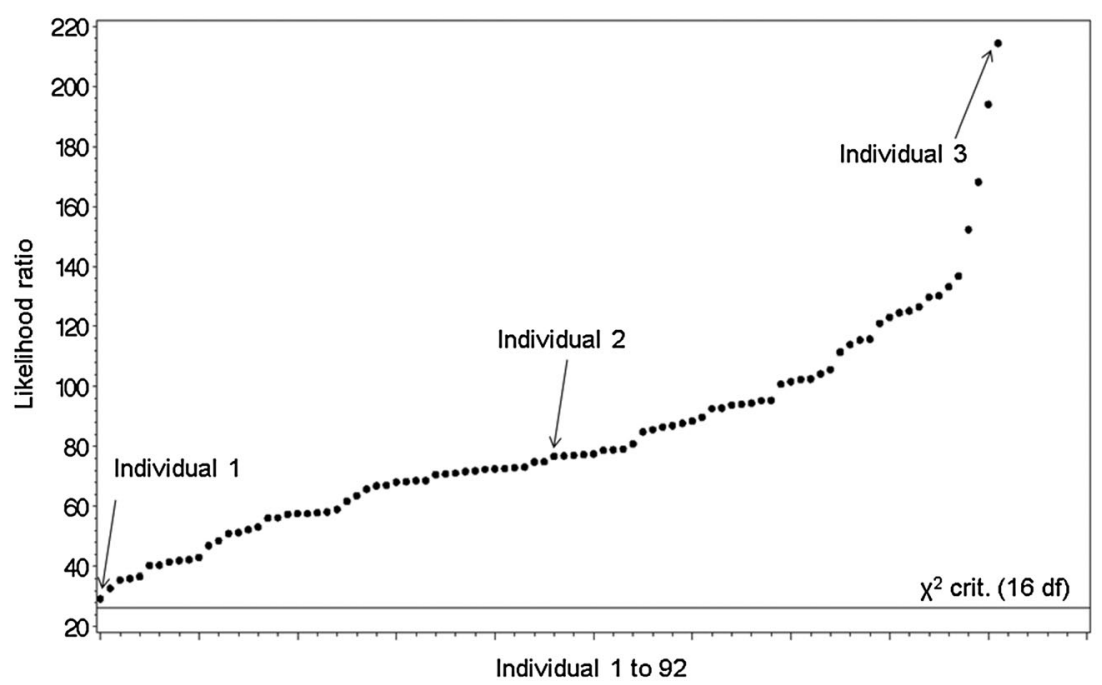

Figure 6. Test of invariance across levels of analysis: 92 comparisons of the $-2 L L$ of Multigroup Model 1 (WP and BP analyses: all parameters estimated) and Multigroup Model 2 (WP and BP analyses: loadings and factor intercorrelations fixed across levels); Individuals 1 to 3 were chosen according to their position in rank order (rank 1, rank 46, and rank 92).

Table 3. Correlations between stable dispositions and within-person characteristics as well as the degree of divergence (the likelihood ratio of the within-between comparison)

\begin{tabular}{|c|c|c|c|c|c|c|}
\hline & & $\begin{array}{l}S D \text { sq NA } \\
\text { loadings }^{\dagger}\end{array}$ & $\begin{array}{l}S D \text { sq PA } \\
\text { loadings }{ }^{\dagger}\end{array}$ & $\begin{array}{l}\text { Correlation } \\
\text { PA-NA }\end{array}$ & $\begin{array}{c}\text { Multigroup } \\
\text { Model 2, LR test }\end{array}$ & $\begin{array}{c}\text { Multigroup } \\
\text { Model 3, LR test }\end{array}$ \\
\hline \multirow[t]{3}{*}{ Personality } & Neuroticism & -.13 & & -.09 & -.11 & .03 \\
\hline & Extraversion & & .03 & & -.004 & \\
\hline & Arousal focus & & .13 & & -.08 & \\
\hline \multirow[t]{10}{*}{ Well-being } & Trait NA & 0.00 & & .06 & $-.22 *$ & .01 \\
\hline & Trait PA & $0.25 *$ & & $.23^{*}$ & .18 & $-.22 *$ \\
\hline & Depressive symptoms, CESD & $-0.28 * *$ & & -.09 & -.11 & .03 \\
\hline & Life satisfaction & 0.06 & & .02 & .04 & .06 \\
\hline & Autonomy & $0.25^{*}$ & & .08 & .06 & -.05 \\
\hline & Environmental mastery & 0.03 & & .18 & .05 & -.15 \\
\hline & Personal growth & $0.23 *$ & & $.24 *$ & .03 & $-.26^{*}$ \\
\hline & Positive relations with others & 0.04 & & .04 & .11 & -.08 \\
\hline & Purpose in life & $0.27 *$ & & .16 & .05 & $-.25^{*}$ \\
\hline & Self-acceptance & 0.01 & & .12 & -.01 & -.12 \\
\hline \multirow{3}{*}{ Stress } & Major life events & -0.18 & & $-.22 *$ & -.06 & .12 \\
\hline & Daily stressors & -0.13 & & -.08 & $-.25^{*}$ & .17 \\
\hline & Perceived stress & -0.19 & & -.02 & $-.25^{*}$ & .08 \\
\hline
\end{tabular}

Note: ${ }^{*} p<.05 ; * * p<.01 ;{ }^{\dagger}$ Correlations are partial correlations, partialing out the loadings' mean; SD sq NA loadings = SD of the squared NA loadings; NA, negative affect; PA, positive affect; CES-D, Center for Epidemiologic Studies-Depression Scale; likelihood ratio test of Multigroup Model 2: loadings, but not the factor intercorrelation, were fixed across levels; likelihood ratio test of Multigroup Model 3: the factor intercorrelation but not loadings were fixed across levels; LR = likelihood ratio.

\section{Associations between the degree of invariance and dispositions}

Closely related to the preceding, we examined whether the degree of within-between divergence (individual differences in the LR) is meaningful from a substantive perspective. We tested (a) whether the degree of divergence is smaller in individuals high in neuroticism, low in well-being and high in enduring stress levels because they were supposed to have more homogeneous NA loadings. Relatedly, we tested (b) whether the degree of divergence is smaller in individuals high in extraversion and arousal focus because they were supposed to have more homogenous PA loadings. Finally, we tested (c) whether the degree of divergence is smaller in individuals high in neuroticism, well-being and enduring stress levels, because these were supposed to have a smaller (more negative) PA-NA factor intercorrelation. Because predictions (a) and (c) go in different directions and pertain to different parts of the model, we conducted two different analyses. First, we repeated the series of multigroup models described above but only fixed the loadings across levels of analyses (Multigroup Model 2). In that way we could test whether the degree of divergence that is due to loading patterns is related to stable dispositions. Next, we repeated the series of multigroup models but only fixed the factor intercorrelation across levels of analyses (Multigroup Model 3 ). Thereby we could test whether the degree of divergence that is due to the factor intercorrelation is related to dispositions. Findings are presented in Table 3. 
The degree of divergence of Multigroup Model 2 (fixed loadings) was not related to personality, but it was related to well-being. The WP structures of individuals with higher levels of trait NA were more similar to the BP affect structure (i.e., the degree of divergence was smaller in individuals high in NA, which resulted in a negative correlation). The other indicators of well-being were not associated with the degree of divergence. In line with our expectations, stress was related to the degree of divergence. The WP structure of individuals with a high level of daily stressors more closely resembled the BP structure (the more stressors, the smaller the LR). Likewise, the WP structures of individuals with high levels in perceived stress more closely resembled the BP structure in terms of loadings patterns.

The degree of divergence of Multigroup Model 3 (fixed factor intercorrelation) was not related to personality, but it was again related to well-being. Individuals with higher levels of trait PA were more similar to the BP affect structure when only the factor intercorrelation was fixed (the degree of divergence is smaller which results in a negative correlation). Likewise, individuals with a higher sense of personal growth and purpose in life had WP structures more similar to the BP structure when only the factor intercorrelation was fixed. Indicators of stress were not correlated with the degree of divergence in Multigroup Model 3.

\section{DISCUSSION}

This study formally tested whether the structure of affective experiences as measured with PANAS items is equivalent when analysed between and within individuals. The answer is unambiguous-findings from the WP level are not equivalent to the BP level. Person-specific analyses revealed that patterns of affect variability across time are person specific, as indicated by individual differences in loading matrices and factor intercorrelations. Importantly, we worked with a restricted NA factor in comparison to the NA subscale of the PANAS because five items did not vary sufficiently across time in multiple individuals. These findings support the notion that BP and WP variation require separate inquiries and that they should not serve as surrogates for each other (Molenaar, 2004). Yet, there were individual differences in the degree of individuals' divergence from the BP structure. Some individuals had factor structures that were more similar to the BP structure, namely those who had more homogeneous NA and PA loadings and a less negative NAPA factor intercorrelations. These characteristics of WP structures were associated with stable attributes of individuals, in particular indicators of well-being. Relatedly, the statistical deviation of WP structures from the BP structure was associated with stable attributes in the domains of well-being and stress. These findings are in line with propositions on links between stable and dynamic aspects of WP functioning (e.g., Mischel \& Shoda, 1998).

\section{Between-person and within-person structures of affect}

Our knowledge about the structure underlying affective experiences is largely determined by cross-sectional studies of BP differences (see, e.g., a recent metaanalysis with more than 250 cross-sectional studies that used the PANAS; Leue \& Lange, 2011). As the PANAS samples a limited range of affective experiences (states high in activation), and as there is consensus about their associations (high correlations within each dimension, low correlations between them; Carroll et al., 1999; Watson \& Clark, 1997), one may be led to think that patterns of WP and BP covariation should resemble each other. Supporting these notions, observational comparisons of WP and BP structures resulted in the conclusion that there are substantial similarities (Zevon \& Tellegen, 1982; see also Merz \& Roesch, 2011).

Despite this limited evidence favouring congruence of BP and WP affect structures, we offered conceptual reasons that speak against the assumption that BP and WP affect structures are equivalent. We noted that the causes of variation between persons differ from the causes of variation within persons, rendering it unlikely that WP affect structures resemble BP structures. In particular, the structure of affect variation at the WP level should largely be determined by situations and by person by situation interactions (Larsen \& Cutler, 1996; Zelenski \& Larsen, 2000; Vansteelandt et al., 2005). Therefore, we expected deviations from the BP structure and emphasized that there should be individual differences in how affect is structured across time. In examining this, we took an idiosyncratic perspective rather than generalizing across subjects (e.g., by investigating an average WP structure). The findings on individual differences in loadings, the heterogeneity of NA and PA loadings, the PA-NA factor intercorrelation and the lack of equivalence between $\mathrm{BP}$ and WP structures all supported our view that BP and WP affect structures are, in fact, different from each other. At the level of individuals, items within the PA and NA dimensions were not necessarily highly correlated. Factor intercorrelations ranged from positive to negative and were on average more negative than the BP correlation.

These findings have important implications and open up new research questions. First and foremost, these findings imply that BP data and analyses do not qualify as a valid surrogate to attain information on affect structures within individuals. It would, for example, simply be wrong to conceive of PA and NA as independent at the WP level. Instead, WP data and analyses are needed to test psychological theory that is in fact often formulated in terms of WP functioning. For example, PA may function as a buffer in times of stress and may ameliorate NA, or PA may diminish in such times while NA becomes overwhelming (Ong et al., 2006; Zautra et al., 2002). Observing independence of PA and NA at the BP level (as is common, see above) can be mistaken as speaking against these hypotheses, which, however, can only be tested by means of WP data and analysis. The findings of this study underscore this point. Affective states cooccurred differently within individuals across time than at a given time across individuals, and the functional WP relationship between PA and NA seems to vary considerably across individuals. This conclusion supports the general plea for a person-specific perspective in psychology, which seeks to assess and model psychological phenomena as they emerge and develop across time within persons (Nesselroade, 1991; cf. Lindenberger \& 
von Oertzen, 2006; Molenaar, 2010). Previous comparisons of $\mathrm{BP}$ and WP structures have reached similar conclusions [e.g., in research on personality (Hamaker et al., 2007); in the field of motivation and cognition (Brose, Schmiedek, Lövdén, Molenaar, \& Lindenberger, 2010); in emotional response patterns between individuals (Hershberger et al., 1994)].

In the long run, the sampling and modelling of time series of affective experiences should lead to a theory of affect variability at the level of individuals (for a seminal step in this direction, see Kuppens et al., 2010). As we will elaborate in the following two sections, such research will need to deal with the essential questions of (a) how individual differences in WP affect structures and in their divergence from the BP structure can be explained and (b) whether the dimensional model of affect at the BP level is appropriate at the WP level.

\section{Divergence of between-structures and within-structures: a matter of degree}

Explaining individual differences in within-between divergences Although we emphasize the necessity to pursue research at the WP and BP level, we do not claim their independence. Instead, we agree with others on the intrinsic relationships that exist between dynamic aspects of psychological functioning and stable dispositions and on the necessity to explain differences in individuals' dynamics (Kuppens, 2009; Mischel \& Shoda, 1998). In this context, we proposed a new line of inquiry for the relationships between WP functioning and BP differences. In particular, we related WP structural characteristics to dispositional and contextual variables that matter for affective experiences (neuroticism, extraversion, well-being and stress). Moreover, the dispositional and contextual variables were related to the degree of divergence of the BP and WP affect structures (i.e., the degree to which a given individual's WP structure deviates from the BP structure (cf., Voelkle et al., 2012).

Along these lines, this study revealed that individuals with a less differentiated affective response (i.e., relatively homogeneous factor loadings; a more negative PA-NA factor intercorrelation) were indeed individuals with lower levels of well-being (in particular, lower levels of trait PA, more depressive symptoms, a lower sense of autonomy, personal growth and purpose in life). These individuals also reported the exposure to a major negative life event more often. Albeit the associations were small and not totally consistent, they are in general in line with the view that the ability to discriminate among emotions and situations is an index of adaptive behaviour (Lindquist \& Barrett, 2008; Mischel \& Shoda, 1998) — a differentiated affective response is positively associated with the proneness to experience positive emotions and positive developmental outcomes (e.g., a sense of autonomy). For these reasons, we see being more similar to the BP structure on the NA loading pattern as less adaptive. To the contrary, life event exposure may result in a reduced ability to differentiate between facets of emotional experiences.

It follows from the preceding that the degree of individuals' divergence from the BP structure should also be related to more stable individual difference characteristics. The data were consistent with this prediction to some degree, but the small size of the correlations has to be kept in mind. The WP affect structures of those individuals with higher levels of stress and trait NA were indeed more similar to the BP structure regarding their loading patterns because they are the ones with more homogeneous loadings. A separate analysis revealed that the WP structures of those individuals with higher levels in measures of well-being (i.e., trait PA, sense of personal growth and purpose in life) were more similar to the BP structure regarding the factor intercorrelation. Thus, the degree of divergence can indeed be meaningfully interpreted here.

It was necessary to examine the degree of BP-WP divergence that emerges when fixing the loading pattern and when fixing the factor intercorrelation separately because the corresponding predictions went in opposite directions. This seems justified because we proceeded on the basis of a priori considerations. Also, the two structural indicators were not significantly correlated; individuals with a large $S D$ of the NA loadings were not the ones with a less negative factor intercorrelation. This finding is important in itself-aspects of adaptive behaviour can be diverse and manifest themselves in different ways in different people. Some manage not to lose sight of positive experiences when experiencing NA, and others have a specific rather than general affective response. 5

Counter to expectations, individuals high in neuroticism did not seem to have a particularly generalized stress response as would be indicated by homogeneous loadings. The limited range of affective experiences that were analysed in this study may have resulted in this deviation from a previous study's results (Ong \& Bergeman, 2004). Also, the heterogeneity of PA loadings did not correlate with dispositional variables. This may be due to the fact that individuals discriminate less between positive states than between negative states more generally (Vansteelandt et al., 2005; Zelenski \& Larsen, 2000).

In summary, individuals' affect structures differ. Yet, our expectation that differences in individuals' structures are systematically related to stable dispositions was partly confirmed. Relatedly, individuals' degree of divergence from the BP structure could be interpreted meaningfully. The results generally conformed to expectations, but the size of the associations was small, on average. These findings require being followed up in future studies.

\section{Dimensional versus discrete models of affect}

It was proposed in the literature that 'state emotion conform to a discrete model and trait emotion to a more dimensional model' (Zelenski \& Larsen, 2000). A discrete model means that distinct emotions are experienced separately and in reaction to the specifics of the situation. According to

${ }^{5}$ From a conceptual point of view, the separation of the two analyses highlights a problematic issue related to the degree of divergence. Moderators of WP structures such as well-being or stress may lead to multiple and at times opposite predictions about the degree of divergence, and in contrast to our example, the corresponding structural aspects may indeed be correlated. In such cases, the degree of divergence cannot be related meaningfully to stable individual differences characteristics. Future studies on the degree of divergence need to bear this in mind. 
dimensional models, affective experiences are structured by a few underlying basic dimensions. Empirical findings seem to support this view (Zelenski \& Larsen, 2000; see also Vansteelandt et al., 2005), although these studies lack strict tests of whether constraining structures to be alike decreases model fit in comparison to unconstrained models. Our study's findings also speak, on average, for a discrete model of affect at the WP level because the size of NA indicators were rather heterogeneous within individuals, and thus, the different affect facets did not necessarily occur simultaneously. In contrast to this, the high and homogeneous NA loadings at the BP level speak for a dimensional model of trait affect.

A more direct approach to the dimensionality at the WP level would be to explore the dimensionality of affect for each individual. We did not do so for two reasons. First, our departure was the well-established two-factor PA-NA model because we wanted to know how much we know about individuals' affective functioning when we take the BP structure as a surrogate. Second, in particular the 10-item NA subscale of the PANAS is not well suited to explore the existence of a discrete affect model because it measures states from five different content domains (distress, anger, fear, guilt and nervousness; Zevon \& Tellegen, 1982) but with too few items to properly distinguish broader categories of affect. The exploration of the dimensionality of individuals' affect structures thus needs to be realized in future studies with a larger set of items - potentially with the shortened version of the Profile of Mood States (Cranford et al., 2006), which shows reliable variability in anxious and depressed mood, anger, fatigue and vigour.

Over and above this, there is probably more than one proper affect model at the WP level. This study revealed large individual differences in loading patterns, some being more heterogeneous and some being more homogeneous. The patterns of the latter individuals may still confirm to a dimensional and not a discrete model (e.g., Figure 5, Individual 1). Thus, an either-or view on whether state emotion conforms to a discrete model and trait emotion conforms to a dimensional model of affect may not do justice to the individual differences of structural characteristics. Just as the BP structure is not informative about WP functioning, a generalized description of WP functioning also fails to do justice to the affect structure of many individuals participating in this study (see also Table 2, columns 2 and 4: these averages may not be indicative for any specific individual's coefficients).

\section{Limitations and future directions}

\section{Removal of items}

The use of only five NA items clearly is a critical issue of this study. Five of the 10 NA items were excluded due to low variance. This limits the comparability of the findings to prior findings on the structure of affect when measured with the PANAS, and it is a threat to construct validity with regard to the NA subscale of the PANAS. At the same time, the finding of particularly low variability on some of the NA items is important in itself. Feeling guilty, ashamed, hostile, scared, and afraid (the excluded items) likely mark relevant emotional moments in people's lives, but these emotional states do not seem to be part of the more general ups and downs of mood in daily life that is multiply determined. Thus, the blend of items that capture more specific and phenomenologically different emotions (guilt, fear) on the one hand and moods (nervousness) on the other hand as found in the PANAS does not seem ideal for measuring affect within individuals across time in diary studies. This may be considered a shortcoming but is also a meaningful insight of this study: WP and BP variation differs to the degree that measures cannot simply be exchanged from one level to the other. This raises the question on how to assess affect in daily life more generally, and some solutions were already provided (Cranford et al., 2006; Wilhelm \& Schoebi, 2007).

\section{Data preprocessing}

Data were z-transformed in this study because seminal work on the structure of affect was also based on the analyses of correlation matrices and because we think it is important that this study's results can be related to prior findings in the literature. Note, however, that the practice of analysing correlation matrices in covariance structure analysis in general and in the analysis of measurement invariance in particular has been criticized for multiple reasons (Cudeck, 1989; Wicherts \& Dolan, 2010). For example, WP variances were person and item specific in this study-z-transforming such data may change the models under investigation.

\section{Removal of participants}

No WP factor solutions were obtained for nine individuals. These individuals had lower mean levels in negative affect across study time. The effect sizes of the mean differences on the other study variables were small to medium, but the pattern was inconclusive. We thus think that the reported results, including the fact that some individuals report so low levels of NA that it prevents determining their structures, are valid for the total sample that was investigated in this study.

\section{Nomothetic generalizations}

How to derive nomothetic generalizations when the starting point are person-specific analyses is one important issue for future research. One way would be to empirically identify groups of individuals whose patterns of covariation are alike (Nesselroade \& Molenaar, 1999) or to identify a group's structure and significant deviations of subgroups and individuals from this structure (Gates \& Molenaar, 2012). Central features at the subgroup level can then be inspected in a second step. In this context, the use of latent class analysis seems promising, as it provides a way for reconciling dimensional, variable-centred approaches with categorical, person-oriented approaches (Loken \& Molenaar, 2008).

\section{Affect dynamics}

Another line of future research should deal with the timedependent, dynamic aspects of WP functioning more comprehensively. This study modelled time-dependent aspects of the time series (lagged and cross-lagged effects) to account for the data's dependencies. These and also other indicators of affective dynamics (e.g., cyclicity; Chow, 
Hamaker, Fuita, \& Boker, 2009; Ram et al., 2005) may be related to the structural parameters of within-person affect variation, and fully understanding the ties that exist between stable dispositions and WP affect functioning will require that WP affect structures are modelled as complexly as necessary. Furthermore, we assumed stationarity of all within-person parameters (i.e., we did not expect trends or shifts in model parameters), and regarding the innovations, we detrended the observed variables to prevent nonstationarity. However, nonstationarity of the affective dynamics is in itself a highly intriguing phenomenon. It may occur, for example, in transition phases (e.g., in adolescence; Molenaar, Sinclair, Rovine, Ram, \& Corneal, 2009), and future studies may benefit from systematically including information of (potentially unnoticed) transition phases.

Affective experiences-a network of causally related states? Throughout this study, we have conceptualized BP and WP facets of affect as manifestations of the latent variables PA and NA that, however, have a different meaning and causes of variation on the different levels of analyses. The network perspective on psychological constructs (Borsboom \& Cramer, 2013) has started puzzling our view on how affective experiences may be conceptualized. According to this view, behavioural components do not require the existence of underlying latent variables. Instead, the components themselves may interact in a causal manner and thereby result in what is labelled at a higher level of abstraction. It seems plausible to also think of affective experiences in terms of systems of causally connected components (e.g., being upset may result in hostility which may reduce interest in social situations), and we think such a conceptualization is well suited to do justice to idiosyncratic forms of behaviours.

\section{Conclusion}

This study has shown that WP and BP structures of affect are not equivalent and that people differ in affect structures and dynamics. Thereby, this study confirms the claim that BP variation must not be used as a surrogate for WP variation (Molenaar, 2004). Instead, BP and WP variation as well as correlates and causes of variation need to be analysed as distinguishable phenomena. The results of this study also revealed that some individuals' affect structures are more similar to the BP affect structure. Individual differences in WP structural characteristics, but also in the degree of their divergence from the BP structure, were associated with stable dispositional and contextual variables, in particular, indicators of well-being and enduring stress. Comparing WP structures on various aspects of affect, personality and well-being among each other and to the corresponding BP structure opens up a new way of thinking about the relationship between interindividual differences and intraindividual development as well as stable and dynamic aspects of personality.

\section{ACKNOWLEDGEMENTS}

The COGITO study was supported by the Max Planck Society, including a grant from Max Planck Society's Innovation Fund
(M.FE.A.BILD0005); the Alexander von Humboldt Foundation's Sofja Kovalevskaja Award (to Martin Lövdén) donated by the German Federal Ministry for Education and Research (BMBF); the German Research Foundation (DFG; KFG 163); and the BMBF (CAI). We thank Conor Dolan for support when applying mkfm6, and Julia Delius for editorial assistance

\section{REFERENCES}

Barrett, L. F., \& Russell, J. A. (1998). Independence and bipolarity in the structure of current affect. Journal of Personality and Social Psychology, 74, 967-984.

Bleidorn, W., \& Peters, A. L. (2011). A multilevel multitraitmultimethod analysis of self- and peer-reported daily affective experiences. European Journal of Personality, 25, 398-408.

Borsboom, D., Mellenbergh, G. J., \& van Heerden, J. (2003). The theoretical status of latent variables. Psychological Review, 110, 203-219.

Borsboom, D., \& Cramer, A. O. J. (2013). Network analysis: An integrative approach to the structure of psychopathology. Annual Review of Clinical Psychology, 9, 91-121.

Brose, A., Schmiedek, F., Lövdén, M., \& Lindenberger, U. (2011). Normal aging dampens the link between intrusive thoughts and negative affect in reaction to daily stressors. Psychology and Aging, 26, 488-502.

Brose, A., Schmiedek, F., Lövdén, M., Molenaar, P. C. M., \& Lindenberger, U. (2010). Adult age differences in covariation of motivation and working memory performance: Contrasting between-person and within-person findings. Research in Human Development, 7, 61-78.

Brose, A., Lindenberger, U., \& Schmiedek, F. (2013). Affective states contribute to trait reports of affective well-being. Emotion, 13, 940-948.

Carroll, J. M., Yik, M. S. M., Russell, J. A., \& Barrett, L. F. (1999). On the psychometric principles of affect. Review of General Psychology, 3, 14-22.

Carstensen, L. L., Mayr, U., Pasupathi, M., \& Nesselroade, J. R. (2000). Emotional experience in everyday life across the adult life span. Journal of Personality and Social Psychology, 79, 644-655.

Chow, S.-M., Hamaker, E. L., Fuita, F., \& Boker, S. M. (2009). Representing time-varying cyclic dynamics using multiplesubject state-space models. British Journal of Mathematical and Statistical Psychology, 62, 683-716.

Cohen, S., Kamarck, T., \& Mermelstein, R. (1983). A global measure of perceived stress. Journal of Health and Social Behavior, 24, 385-396.

Costa, P. T., \& McCrae, C. S. (1992). The Revised NEO Personality Inventory (NEO PI-R) professional manual. Odessa, FL: Psychological Assessment Resources.

Cranford, J. A., Shrout, P. E., Iida, M., Rafaeli, E., Yip, T., \& Bolger, N. (2006). A procedure for evaluating sensitivity to within-person change: Can mood measures in diary studies detect change reliably? Personality and Social Psychology Bulletin, 32, 917-929.

Crawford, J. R., \& Henry, J. D. (2004). The Positive and Negative Affect Schedule (PANAS): Construct validity, measurement properties and normative data in a large non-clinical sample. British Journal of Clinical Psychology, 43, 245-265.

Cudeck, R. (1989). Analysis of correlation matrices using covariance structure models. Psychological Bulletin, 105, 317-327.

Diener, E., Emmons, R. A., Larsen, R. J., \& Griffin, S. (1985). The satisfaction with life scale. Journal of Personality Assessment, 49, 71-75.

Diener, E., \& Emmons, R. A. (1984). The independence of positive and negative affect. Journal of Personality and Social Psychology, 47, 1105-1117. 
Dolan, C. V. (2010). MKFM6: Multi-group, multi-subject stationary time series modeling based on the Kalman filter. University of Amsterdam [computer software]

Feldman, L. A. (1995). Valence focus and arousal focus: Individual differences in the structure of affective experience. Journal of Personality and Social Psychology, 69, 153-166.

Gates, K. M., \& Molenaar, P. C. M. (2012). Group search algorithm recovers effective connectivity maps for individuals in homogeneous and heterogeneous samples. NeuroImage, 63, 310-319.

Hamaker, E. L., Nesselroade, J. R., \& Molenaar, P. C. M. (2007). The integrated trait-state model. Journal of Research in Personality, 41, 295-315.

Hershberger, S. L., Corneal, S. E., \& Molenaar, P. C. M. (1994). Dynamic factor analysis: An application to emotional response patterns underlying daughter/father and stepdaughter/stepfather relationships. Journal Structural Equation Modeling, 2, 31-52.

Hobson, C. J., Kamen, J., Szostek, J., Nethercut, C. M., Tiedmann, J. W., \& Wojnarowicz, S. (1998). Stressful life events: A revision and update of the social readjustment rating scale. International Journal of Stress Management, 5, 1-23.

Kuppens, P. (2008). Individual differences in the relationship between pleasure and arousal. Journal of Research in Personality, 42, 1053-1059.

Kuppens, P. (2009). The legacy of the person-situation debate for understanding variability in emotional experience. Journal of Research in Personality, 43, 255-256.

Kuppens, P., Oravecz, Z., \& Tuerlinckx, F. (2010). Feelings change: Accounting for individual differences in the temporal dynamics of affect. Journal of Personality and Social Psychology, 99, 1042-1060.

Larsen, R. J., \& Cutler, S. E. (1996). The complexity of individual emotional lives: A within-subject analysis of affect structure. Journal of Social and Clinical Psychology, 15, 206-230.

Larsen, R. J., Diener, E., \& Emmons, R. A. (1986). Affect intensity and reactions to daily life events. Journal of Personality and Social Psychology, 51, 803-814.

Lebo, M. A., \& Nesselroade, J. R. (1978). Intraindividual differences dimensions of mood change during pregnancy identified in five P-technique factor analyses. Journal of Research in Personality, 12, 205-224.

Leue, A., \& Lange, S. (2011). Reliability generalization: An examination of the Positive Affect and Negative Affect Schedule. Assessment, 18, 487-501.

Lindenberger, U., \& von Oertzen, T. (2006). Variability in cognitive aging: From taxonomy to theory. In E. Bialystok, \& I. M. Craik (Eds.), Lifespan cognition: Mechanisms of change (pp. 279-314). Oxford, UK: Oxford University Press.

Lindquist, K., \& Barrett, L. F. (2008). Emotional complexity. In M. Lewis, J. M. Haviland-Jones, \& L. F. Barrett (Eds.), The handbook of emotion (pp. 513-530). New York: Guilford. http://www.amazon. com/Handbook-Emotions-Third-Michael-Lewis/dp/1593856504/

Loken, E., \& Molenaar, P. C. M. (2008). Categories or continua? The correspondence between mixture models and factor models. In G. R. Hancock ,\& K. M. Samuelsen (Eds.), Advances in latent variable mixture models (pp. 277-297). Charlotte, NC: Information Age Publishing.

Merz, E. L., \& Roesch, S. C. (2011). Modeling trait and state variation using multilevel factor analysis with PANAS daily diary data. Journal of Research in Personality, 45, 2-9.

Hautzinger, M. (1988). Die CES-D Skala. Ein Depressionsmeßinstrument für Untersuchungen in der Allgemeinbevölkerung [The CES-D scale: A depression measure for studies of the general population]. Diagnostica, 31, 167-173.

Mischel, W., \& Shoda, Y. (1998). Reconciling processing dynamics and personality dispositions. Annual Review of Psychology, 49, 229-258.

Molenaar, P. C. M. (1985). A dynamic factor model for the analysis of multivariate time series. Psychometrika, 50, 181-202.

Molenaar, P. C. M. (2004). A manifesto on psychology as idiographic science: Bringing the person back into scientific psychology, this time forever. Measurement, 2, 201-218.
Molenaar, P. C. M., Sinclair, K. O., Rovine, M. J., Ram, N., \& Corneal, S. E. (2009). Analyzing developmental processes on an individual level using nonstationary time series modeling. Developmental Psychology, 45, 260-271.

Molenaar, P. C. M. (2010). Testing all six person-oriented principles in dynamic factor analysis. Development and Psychopathology, 22, 255-259.

Muthén, B. O. (1994). Multilevel covariance structure analysis. Sociological Methods Research, 22, 376-398.

Nesselroade, J. R. (1991). The warp and the woof of the developmental fabric. In R. M. Downs, L. S. Liben, \& D. S. Palermo (Eds.), Visions of aesthetics, the environment and development: The legacy of Joachim F. Wohlwill (pp. 213-240). Hillsdale, NJ: Erlbaum.

Nesselroade, J. R., \& Molenaar, P. C. M. (1999). Pooling lagged covariance structures based on short, multivariate time series for dynamic factor analysis. In R. H. Hoyle (Eds.), Statistical strategies for small sample research (pp. 223-250). Thousand Oaks, CA: Sage.

Nesselroade, J. R., McArdle, J. J., Aggen, S. H., \& Meyers, J. M. (2002). Dynamic factor analysis models for representing process in multivariate time-series. In D. S. Moskowitz, \& S. L. Hershberger (Eds.), Modeling intraindividual variability with repeated measures data: Methods and applications (pp. 235-264). Mahwah, NJ: Erlbaum.

Ong, A. D., \& Bergeman, C. S. (2004). Emotional complexity in later life. Journal of Gerontology: Psychological Science, 59, P117-P122.

Ong, A. D., Bergeman, C. S., Bisconti, T. L., \& Wallace, K. A. (2006). Psychological resilience, positive emotions, and successful adaptation to stress in later life. Journal of Personality and Social Psychology, 91, 730-749.

Ostendorf, F., \& Angleitner, A. (2004). NEO-PI-R. NEO Persönlichkeitsinventar nach Costa und McCrae - Revidierte Fassung [Revised NEO Personality Inventory]. Göttingen, Germany: Hogrefe.

Ram, N., Chow, S. M., Bowles, R. P., Wang, L. J., Grimm, K., Fujita, F., \& Nesselroade, J. R. (2005). Examining interindividual differences in cyclicity of pleasant and unpleasant affects using spectral analysis and item response modeling. Psychometrika, 70, 773-790.

Russell, J. A. (1980). A circumplex model of affect. Journal of Personality and Social Psychology, 39, 1161-1178.

Ryff, C. D. (1989). Happiness is everything, or is it: Explorations on the meaning of psychological well-being. Journal of Personality and Social Psychology, 57, 1069-1081.

Schmiedek, F., Bauer, C., Lövdén, M., Brose, A., \& Lindenberger, U. (2010): Cognitive enrichment in old age: Web-based training programs. GeroPsych, 23, 59-67.

Schmukle, S. C., Egloff, B., \& Burns, L. R. (2002). The relationship between positive and negative affect in the Positive and Negative Affect Schedule. Journal of Research in Personality, 36, 463-475.

van Eck, M., Nicolson, N. A., \& Berkhof, J. (1998). Effects of stressful daily events on mood states: Relationship to global perceived stress. Journal of Personality and Social Psychology, $75,1572-1585$.

Vansteelandt, K., Van Mechelen, I., \& Nezlek, J. B. (2005). The co-occurrence of emotions in daily life: A multilevel approach. Journal of Research in Personality, 39, 325-335.

Voelkle, M. C., Brose, A., Schmiedek, F., \& Lindenberger, U. (in press). Towards a unified framework for the study of betweenperson and within-person structures: Building a bridge between two research paradigms. Multivariate Behavioral Research.

Voelkle, M. C., Oud, J. H. L., von Oertzen, T., \& Lindenberger, U. (2012). Maximum likelihood dynamic factor modeling for arbitrary $\mathrm{N}$ and $\mathrm{T}$ using SEM. Structural Equation Modeling, 19, 329-350.

Watson, D. (1988). Intraindividual and interindividual analyses of positive and negative affect: Their relation to health complaints, 
perceived stress, and daily activities. Journal of Personality and Social Psychology, 54, 1020-1030.

Watson, D., \& Clark, L. A. (1992). On traits and temperament: General and specific factors of emotional experience and their relation to the 5-factor model. Journal of Personality, 60, 441-476.

Watson, D., \& Clark, L. A. (1997). Measurement and mismeasurement of mood: Recurrent and emergent issues. Journal of Personality Assessment, 68, 267-296.

Watson, D., Clark, L. A., \& Tellegen, A. (1988). Development and validation of brief measures of positive and negative affect: The PANAS scales. Journal of Personality and Social Psychology, 54, 1063-1070.

Wessman, A. E., \& Ricks, D. F. (1966). Mood and personality. New York: Holt, Rinehart and Winston.

Wicherts, J. M., \& Dolan, C. V. (2010). Measurement invariance in confirmatory factor analysis. An illustration using IQ test performance of minorities. Educational Measurement: Issues \& Practice, 29, 39-47.

Wilhelm, P., \& Schoebi, D. (2007). Assessing mood in daily life: Structural validity, sensitivity to change, and reliability of a short scale to measure three basic dimensions of mood. European Journal of Psychological Assessment, 23, 258-267.

Zautra, A. J., Berkhof, J., \& Nicolson, N. A. (2002). Changes in affect interrelations as a function of stressful events. Cognition \& Emotion, 16, 309-318.

Zelenski, J. M., \& Larsen, R. J. (2000). The distribution of basic emotions in everyday life: A state and trait perspective from experience sampling data. Journal of Research in Personality, 34, 178-197.

Zevon, M. A., \& Tellegen, A. (1982). The structure of mood change: An idiographic/nomothetic analysis. Journal of Personality and Social Psychology, 43, 111-122. 